\title{
Shape Preserving Digitization of Binary Images After Blurring
}

\author{
Peer Stelldinger and Ullrich Köthe \\ Cognitive Systems Group, University of Hamburg, \\ Vogt-Köln-Str. 30, D-22527 Hamburg, Germany
}

\begin{abstract}
Topology is a fundamental property of shapes in pictures. Since the input for any image analysis algorithm is a digital image, which does not need to have the same topological characteristics as the imaged real world, it is important to know, which shapes can be digitized without topological changes. Most existing approaches do not take into account the unavoidable blurring in real image acquisition systems or use extremely simplified and thus unrealistic models of digitization with blurring. In case of the mostly used square grids we show which binary images can be digitized topologically correctly after blurring with an arbitrary non-negative radially symmetric point spread function, which is an important step forward to real digitization.
\end{abstract}

\section{Introduction}

A reliable image analysis algorithm requires a digital image having as many properties as possible in common with its continuous preimage. One intrinsically twodimensional property is the topology of shapes. There are several sampling theorems known, which describe under which circumstances the topology of some shape does not change during digitization. These theorems mostly differ in the chosen digitization model and the used sampling grid. E.g. Pavlidis showed that so-called $r$-regular shapes can be digitized with square grids without any change in topology [4. Serra proved the same for hexagonal grids [6] and recently we extended these results to arbitrary sampling grids [1]. All of these approaches used the subset digitization where a sampling point is set if and only if it lies within the foreground region of the binary image, i.e. no blurring occurs. Unfortunately, real optical systems blur the binary image before the light reaches the optical sensors. In addition to that each sensor integrates the intensity of light over some area. Both effects can be described as blurring - a convolution of the ideal binary image with a suitable point spread function. A binary image can be recovered by considering a particular level set $L_{l}=\left\{x \in \mathbb{R}^{2} \mid \hat{f}(x) \geq l\right\}$ of the blurred image $\hat{f}$, i.e. by thresholding. Of course the resulting shape heavily depends on the choice of the used point spread function. Latecki et al. 2, 3] used a point spread function which is constant in its square-shaped support and proved that $r$-regular images can be topologically correctly reconstructed after blurring and sampling with a sufficiently dense square grid. In the above mentioned previous paper [1] we proved that this is also true for point spread functions, which 


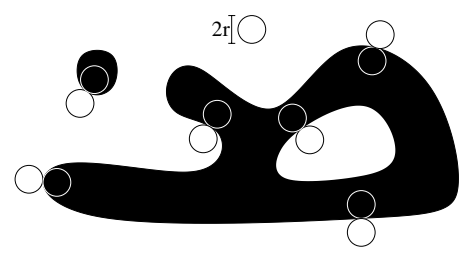

Fig. 1. For each boundary point of an $r$-regular set there exists an outside and an inside osculating open disc of radius $r$

are constant in their disc-shaped support. Up to now nothing has been known about blurring with point spread functions which are not constant within their support. Now we extent the results to arbitrary non-negative radially symmetric point spread functions with bounded support. We prove that given such a point spread function with a support of radius $p$ and a square grid of sampling density $r^{\prime}>p$, every $r$-regular image $\left(r>r^{\prime}+p\right)$ will be digitized whithout any change in the topology.

\section{Regular Sets, Sampling and Reconstruction}

At first we define some basic mathematical concepts. The Complement of a set $A$ will be noted as $A^{c}$. The boundary $\partial A$ is the set of all common accumulation points of $A$ and $A^{c}$. A set $A$ is open, if it does not intersect its boundary. $\mathcal{B}_{r}(c):=\left\{x \in \mathbb{R}^{2} \mid d(x, c) \leq r\right\}$ denotes the closed disc and $\mathcal{B}_{r}^{0}(c):=\left(\mathcal{B}_{r}(c)\right)^{0}$ denotes the open disc of radius $r$ and center $c$. If a point $x$ has the coordinates $x_{1}, x_{2}$, we write $\left(x_{1} \mid x_{2}\right)$ alternatively for $x$. We denote the Euclidean distance between two points $x, y$ as $d(x, y)$ and the Hausdorff distance between two sets $A, B$ as $d_{H}(A, B)=\max \left(\max _{x \in A} \min _{y \in B} d(x, y), \max _{y \in B} \min _{x \in A} d(x, y)\right)$. The dilation of a set $A$ with a disc $\mathcal{B}_{r}$ is defined as $A \oplus \mathcal{B}_{r}:=\left\{x \in \mathbb{R}^{2} \mid d_{H}(A,\{x\}) \leq r\right\}$ and the erosion is $A \ominus \mathcal{B}_{r}:=\left\{x \in \mathbb{R}^{2} \mid d_{H}\left(A^{c},\{x\}\right)>r\right\} . L_{t}(f)$ shall be the level set with threshold value $t$ of an image function $f: \mathbb{R}^{2} \rightarrow \mathbb{R}: L_{t}(f):=\{x \in$ $\left.\mathbb{R}^{2} \mid f(x) \geq t\right\}$.

Most of the existing topological sampling theorems require the binary images to be $r$-regular [1,2, 3, 6, 7,. The concept of $r$-regular images was introduced independently by Serra [6] and Pavlidis [4. These sets are extremely well behaved - they are smooth, round and do not have any cusps.

Definition 1. A compact set $A \subset \mathbb{R}^{2}$ is called $r$-regular if for each boundary point of $A$ it is possible to find two osculating open discs of radius $r$, one lying entirely in $A$ and the other lying entirely in $A^{c}$ (see Fig. 11).

In order to compare analog with digital images, two things are needed: First a method to compare binary images and second a formal description of the processes of sampling and reconstruction. The method for comparison we choose is weak r-similarity (see [1,7]). If two sets are weakly $r$-similar, they are topologically equivalent (this criterion was chosen by Pavlidis [4]), have the same 


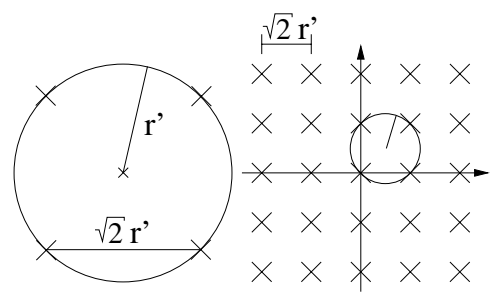

Fig. 2. A square grid with a distance of $\sqrt{2} r^{\prime}$ between two adjacent sampling points is an $r^{\prime}$-grid

homotopy tree (as used by Serra 6]) and a Hausdorff distance of at most $r$. Note that topological equivalence and identity of homotopy trees are different criteria and neither implies the other (see [1]). The usefullness of a bounded Hausdorff distance as similarity criterion is extensively discussed in the work of Ronse and Tajine (see 5 for a summary). The generality of their approach is remarkable, but it cannot directly be used for our problem, since it doesn't say anything about the topology of a digital reconstruction and it cannot be applied to images which are blurred by some point spread function.

Definition 2. Two bounded sets $A, B \subset \mathbb{R}^{2}$ are called weakly $r$-similar if there exists a homeomorphism $f: \mathbb{R}^{2} \rightarrow \mathbb{R}^{2}$ such that $x \in A \Leftrightarrow f(x) \in B$, and the Hausdorff distance between the set boundaries $d_{H}(\partial A, \partial B) \leq r \in \mathbb{R}_{+} \cup\{\infty\}$. The used homeomorphism is called $\mathbb{R}^{2}$-homeomorphism between $A$ and $B$.

In most practical cases the sampling grid is a square grid, as used in several previous sampling theorems [2, 3, 4. In this paper we will restrict ourselves to this kind of sampling grid, although we used a more general approach in previous papers [1,7]. The reason is that the restriction to square grids allows us to prove a sampling theorem for a much wider class of point spread functions.

Definition 3. A countable set $S \subset \mathbb{R}^{2}$ of sampling points with $d_{H}\left(S, \mathbb{R}^{2}\right) \leq r^{\prime}$, i.e. the Euclidean distance from each point $x \in \mathbb{R}^{2}$ to the next sampling point is at most $r^{\prime} \in \mathbb{R}$, is called an $r^{\prime}$-grid if $S \cap A$ is finite for any bounded set $A \in \mathbb{R}^{2}$. The pixel $\operatorname{Pixel}_{S}(s)$ of a sampling point $s$ is its Voronoi region, i.e. the set of all points lying at least as near to this point as to any other sampling point. If $S=a \cdot R \cdot \mathbb{Z}^{2}+b$ for some constant $a \in\left(0, \sqrt{2} r^{\prime}\right]$, rotation matrix $R$ and vector $b \in \mathbb{R}^{2}, S$ is called square grid (see Fig. 2). The union of the pixels with sampling points lying in $A$ is the reconstruction of $A$ w.r.t. $S$, also called the $S$-reconstruction of $A: \quad \hat{A}:=\bigcup_{s \in S \cap A} \operatorname{Pixel}_{S}(s)$. Two pixels are adjacent if they share an edge. Two pixels of $\hat{A}$ are connected if there exists a chain of adjacent pixels in $\hat{A}$ between them. Two sampling points are adjacent (connected) if their pixels are adjacent (connected). A component of $\hat{A}$ is a maximal set of connected pixels.

This most obvious approach for sampling is to restrict the domain of the image function to the sampling grid. But this ideal digitization does not take 


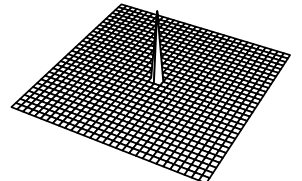

(a)

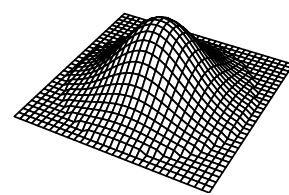

(d)

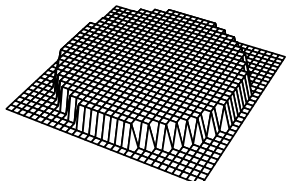

(b)

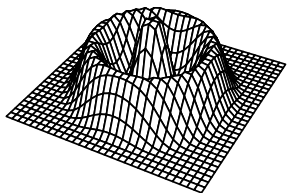

(e)

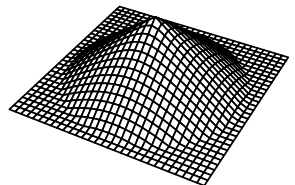

(c)

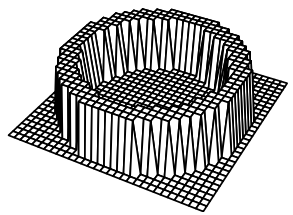

(f)

Fig. 3. The definition of $p$-PSFs is very broad. Examples are the dirac impulse (a), which leads to a non-blurred digitization, the disc-PSF (b) as used in 17], conic PSFs (c), truncated Gaussians (d), and even non-descending PSFs (e) and (f). While (f) is an artificial example showing what kind of PSF is also allowed, (e) is of practical interest, since the camera aperture can cause such diffraction patterns

into account any blurring. This can be added by a convolution of the image with some point spread function before sampling. Digitization of a binary image has three steps: At first the image gets blurred due to the camera optic. Then the blurred grayscale preimage gets sampled and reconstructed (To reconstruct a grayscale image means to fill each pixel with the image value at the corresponding sampling point). Finally the image gets thresholded in order to get a binary result. Mathematically the last two steps commute. Thus the definition of a digitization without blurring completely determines how to digitize with some blurring. You simply have to blur the original set, apply a threshold function and digitize the result.

Definition 4. A function $k: \mathbb{R}^{2} \longrightarrow \mathbb{R}$ is called a point spread function (PSF) if $\int_{\mathbb{R}^{2}} k(x) d x=1$. The PSF $k$ is a blurring PSF if it is non-negative. The PSF $k_{f}$ with $k_{f}(x):=f(|x|)$ for some function $f: \mathbb{R}_{+} \longrightarrow \mathbb{R}$ with $\int_{0}^{\infty} r \cdot f(r) d r=\frac{1}{2 \pi}$ is called radially symmetric. The function $f$ is called the generator function of $k_{f}$. If $k_{f}$ is a radially symmetric blurring PSF with $f(r)=0$ for every $r$ greater than some $p$, it is called a p-PSF. Now let $A \subset \mathbb{R}^{2}$ be a binary set. Then its characteristic function $\chi_{A}: \mathbb{R}^{2} \rightarrow\{0,1\}$ is 1 for any $x \in A$ and 0 for any $x \notin A$. Given a PSF $k$, the blurred image of $A$ by using $k$ is defined as $f_{A}:=k \star \chi_{A}$ ( denotes convolution).

With these definitions we have everything we need to prove a sampling theorem for blurred binary images. 

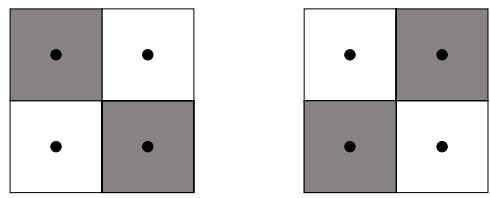

Fig. 4. After topologically correct digitization with a square grid none of the shown configurations can occur. Thus the resulting image is well-composed

\section{Sampling-Theorem for Blurred Binary Images}

In a previous paper we already proved a sampling theorem for non-blurred binary images and a theorem for binary images after blurring with a constant discshaped PSF [1]:

Theorem 1. Let $r \in \mathbb{R}_{+}$and $A$ an $r$-regular set. Then $A$ is weakly $r^{\prime}$-similar to any $S$-reconstruction with some $r^{\prime}$-grid $S, 0<r^{\prime}<r$.

In case of square grids this implies that the $S$-reconstruction of an $r$-regular set is well-composed, as defined by Latecki (see [3]). This means, the digital image does not contain any of the pixel configurations shown in Fig. 4

Theorem 2. Let $r, r^{\prime}, p \in \mathbb{R}_{+}$be positive numbers with $r^{\prime}+p<r$ and let $A$ be an $r$-regular set, $k_{p}$ a $p$-PSF, and $f_{A}=k_{p} \star \chi_{A}$ the blurred image of $A$. Further let $L_{l}$ be the level set of $f_{A}$ with some level $l$ and let $S$ be an arbitrary $r^{\prime}$-. Then the $S$-reconstruction $\hat{L}_{l}$ of $L_{l}$ is weakly $\left(r^{\prime}+p\right)$-similar to $A$.

In order to generalize these results to other types of point spread functions we restricted ourselves to square grids. By doing this we are able to show that any $p$-PSF with $p<r^{\prime}$ can be used for digitization with an $r^{\prime}$-grid, such that any $r$-regular set $\left(r>r^{\prime}+p\right)$ is topologically equivalent to its digital reconstruction:

Theorem 3. Let $r, r^{\prime}, p \in \mathbb{R}_{+}$be positive numbers with $p<r^{\prime}$ and $r^{\prime}+p<r$ and let $A$ be an $r$-regular set, $k_{p}$ an arbitrary $p-P S F$, and $f_{A}=k_{p} \star \chi_{A}$ the blurred image of $A$. Further let $L_{l}$ be the level set of $f_{A}$ with some level $l$ and let $S$ be a square grid, which is an $r^{\prime}$-grid. Then the $S$-reconstruction $\hat{L}_{l}$ of $L_{l}$ is weakly $\left(r^{\prime}+p\right)$-similar to $A$.

Proof. With $r^{\prime}>p$ follows $2 r^{\prime}>r^{\prime}+p$. Now let $s \in \mathbb{R}_{+}$be any number in the interval $\left(r^{\prime}+p, 2 r^{\prime}\right]$. Then $A$ is $s$-regular. If we can prove the theorem for such an $s$ instead of $r$, it is also true for $r>s$. We make use of the inequality $s \leq 2 r^{\prime}$ below.

Due to the support of the PSF, $f_{A}(x)=1$ for any $x \in A \ominus \mathcal{B}_{p}$ and analogously $f_{A}(x)=0$ for any $x \notin A \oplus \mathcal{B}_{p}$. Due to $s$-regularity of $A$, the sets $B:=A \ominus \mathcal{B}_{p}$ and $C:=A \oplus \mathcal{B}_{p}$ are both $(s-p)$-regular and weakly $p$-similar to $A$. Due to Theorem 1 their $S$-reconstructions $\hat{A}, \hat{B}$ are weakly $\left(r^{\prime}+p\right)$-similar to $A$. Obviously $\hat{B} \subseteq \hat{L}_{l} \subseteq \hat{C}$, which implies that the Hausdorff-distance between 


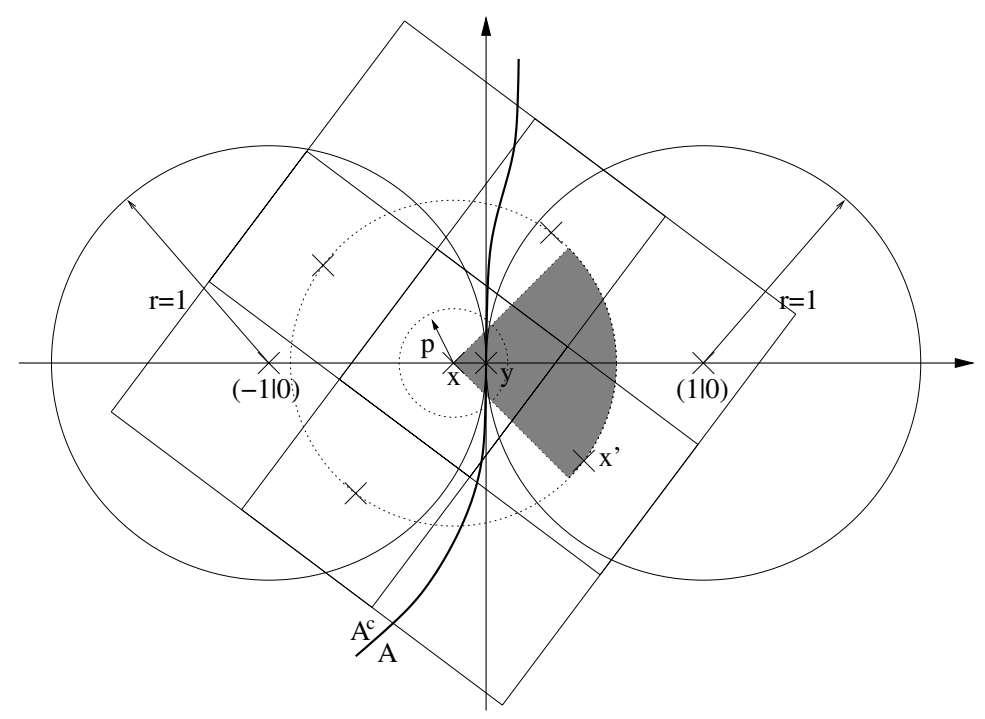

Fig. 5. At least one of the sampling points being adjacent to $x$ lies in the shaded sector

$\partial A$ and $\partial \hat{L}_{l}$ is bounded by $r^{\prime}+p$. Thus we only have to show that $L_{l}$ is $\mathbb{R}^{2}$ homeomorphic to $A$. This is the case if any sampling point $x \in \hat{L}_{l}$ is (directly) connected in $\hat{L}_{l}$ with some sampling point $y \in \hat{B}$ and if any sampling point $x \notin \hat{L}_{l}$ is connected in $\hat{L}_{l}^{c}$ with some sampling point $y \notin \hat{C}$, because then no additional component or hole can occur. We show this by proving that for any $x \in S$ with $f_{A}(x) \in(0,1)$ there exists an adjacent sampling point $x_{>}$with $f_{A}\left(x_{\geq}\right) \geq f_{A}(x)$ and an adjacent sampling point $x_{\leq}$with $f_{A}\left(x_{\leq}\right) \leq f_{A}(x)$. This implies that the configurations shown in Fig. 团 cannot occur.

Let $x \in S$ be a sampling point with $f_{A}(x) \in(0,1)$ and let $y \in \partial A$ be the boundary point of $A$ being nearest to $x$. Due to $s$-regularity there exists a unique nearest boundary point. Without loss of generality let $x=(d \mid 0), y=(0 \mid 0), s=1$ (any other case can be derived by choosing an appropriate scale and coordinate system) and let $\mathcal{B}_{1}^{0}((1 \mid 0))$ be the inside and $\mathcal{B}_{1}^{0}((-1 \mid 0))$ be the outside osculating $s$-disc of $A$ in $y$ (see Fig. (5). Then the four sampling points being adjacent to $x$ lie on the circle with radius $\sqrt{2} r^{\prime}$ and center $x$. At least one of them, which will be noted as $x^{\prime}$, lies on the rightmost quarter circle which is bounded by the points $\left(d+r^{\prime} \mid r^{\prime}\right)$ and $\left(d+r^{\prime} \mid-r^{\prime}\right)$ (see Fig. (5). Now let $D_{1}=1+d$ be the distance between $(-1 \mid 0)$ and $x$, let $D_{2}=\sqrt{\left(1-r^{\prime}-d\right)^{2}+r^{\prime 2}}$ be the distance between $(1 \mid 0)$ and $\left(d+r^{\prime} \mid r^{\prime}\right)$ and let $D_{3}$ be the distance between (1|0) and $x^{\prime}$ (see Fig. 6). Then $D 3 \leq D_{2}$ since the center $(d \mid 0)$ of the circle containing $x^{\prime}$ is to the left of $(1 \mid 0)$.

Now let $B:=\mathcal{B}_{1}^{c}$ be a binary image, which is the complement of the unit disc. By using $B$ we construct a helper function $h:[1-p, 1+p] \longrightarrow[0,1]$ with $h(z):=f_{B}((z \mid 0))$ (see Fig. (7). Obviously $h$ is monotonically increasing since the non-zero area $\mathcal{B}_{p}\left(\left(z_{1} \mid 0\right)\right) \cap B$ of the image $B$ covered by the PSF at postion $\left(z_{1} \mid 0\right)$ is a translated superset of the same area $\mathcal{B}_{p}\left(\left(z_{2} \mid 0\right)\right) \cap B$ at position $\left(z_{2} \mid 0\right)$ for 


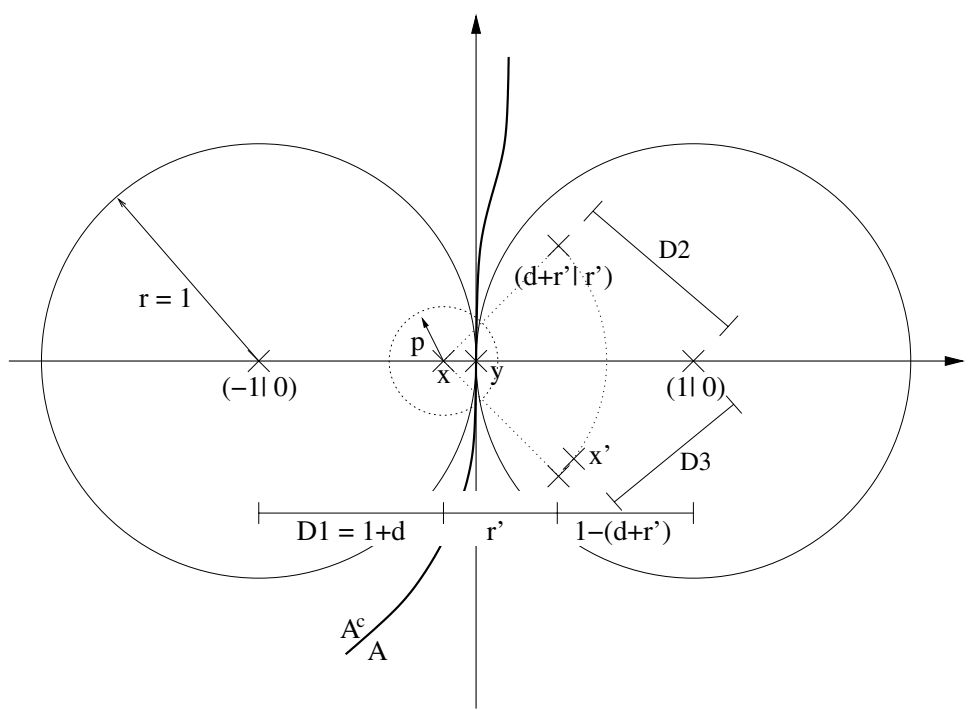

Fig. 6. The distance $D 3$ between the center of the inside osculating disc and the adjacent sampling point $x^{\prime}$ is at most equal to the distance $D_{2}$

any $z_{1}, z_{2} \in[1-p, 1+p]$ with $z_{1}>z_{2}$. The two circles $\partial \mathcal{B}_{1}$ and $\partial \mathcal{B}_{p}((z \mid 0))$ have at most two points in common. There exists exactly one other circle of radius 1 sharing these points. This circle is centered in $\left(\frac{1-p^{2}}{z}+z \mid 0\right)$. As Fig. 7 illustrates, $1-h(z) \geq h\left(\frac{1-p^{2}}{z}\right)$.

Since the outside osculating disc $\mathcal{B}_{1}((-1 \mid 0))$ is a subset of $A^{c}$ (see Fig. 5), $f_{A}(x)$ is at most equal to $h\left(D_{1}\right)$. Analogously since the inside osculating $\operatorname{disc} \mathcal{B}_{1}((1 \mid 0))$ is a subset of $A, f_{A}\left(x^{\prime}\right)$ is at least equal to $1-h\left(D_{3}\right)$. With $D_{3} \leq D_{2}$ it follows that $1-h\left(D_{2}\right) \leq 1-h\left(D_{3}\right)$. Thus we only have to show that $h\left(D_{1}\right) \leq 1-h\left(D_{2}\right)$ in order to prove $f_{A}(x) \leq f_{A}\left(x^{\prime}\right)$.

We know that $-p \leq d \leq p$ and $0 \leq 1-r^{\prime} \leq \frac{1}{2}$ (because $1=s \leq 2 r^{\prime}$ ). Now suppose to the contrary, $h\left(D_{1}\right)>1-h\left(D_{2}\right)$. Then $h\left(D_{1}\right)>h\left(\frac{1-p^{2}}{D_{2}}\right)$ and due to montony of $h$ follows $D_{1}>\frac{1-p^{2}}{D_{2}}>\frac{1-\left(1-r^{\prime}\right)^{2}}{D_{2}}$. By substitution of $D_{1}$ and $D_{2}$ we get $1+d>\frac{1-\left(1-r^{\prime}\right)^{2}}{\sqrt{\left(1-r^{\prime}-d\right)^{2}+r^{\prime 2}}}$. Since both sides of the inequation are positive, we can square it, and further simplification leads to

$$
\frac{\left(1-r^{\prime}\right)-d}{(1+d)^{2}}\left(2+2 d+2 d^{2}+d^{3}-(2+d)^{2}\left(1-r^{\prime}\right)+d\left(1-r^{\prime}\right)^{2}+\left(1-r^{\prime}\right)^{3}\right)<0 .
$$

Since the fraction is always non-negative for the allowed $d, r^{\prime}$, we only have to look at the rest of the inequation. This inequation is equivalent to both of the following inequations: 


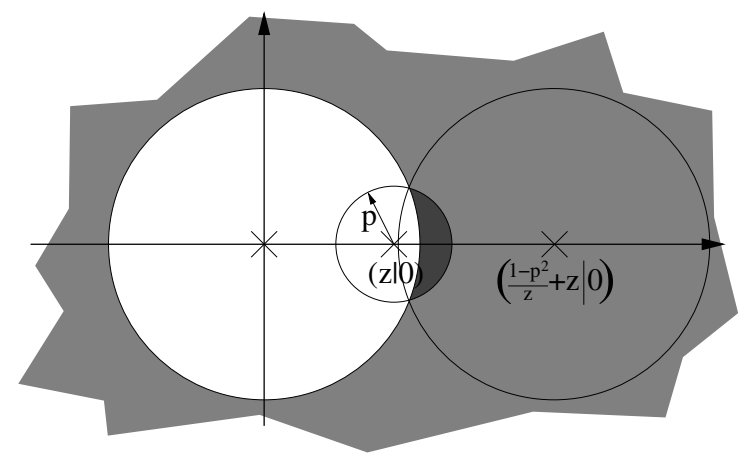

Fig. 7. The helper function $h$ describes the result of blurring the complement of the unit disc image with the PSF at some position with distance $z$ to the origin

$$
\begin{gathered}
2(1+d)\left(1-2\left(1-r^{\prime}\right)\right)+\left(1-r^{\prime}\right)\left(\left(1-r^{\prime}\right)+d\right)^{2}+ \\
2 d^{2}\left(1-\left(1-r^{\prime}\right)\right)+(-d)\left(\left(1-r^{\prime}\right)^{2}-d^{2}\right)<0 \\
2(1+d)\left(1-2\left(1-r^{\prime}\right)\right)+d\left(1-r^{\prime}\right)\left(\left(1-r^{\prime}\right)-d\right)+ \\
d^{3}+\left(1-r^{\prime}\right)^{3}+2 d^{2}<0
\end{gathered}
$$

In case of $d<0$ each addend of the first inequation consists completely of nonnegative factors and in case of $d \geq 0$ each addend of the second inequation consists completely of nonnegative factors for $|d| \leq p<1-r^{\prime} \leq \frac{1}{2}$. Thus for any $d$ one of the inequations is obviously false which implies that the assumption $h\left(D_{1}\right)>1-h\left(D_{2}\right)$ is not true. It follows that for any $x \in S$ with $f_{A}(x) \in(0,1)$ there exists an adjacent sampling point $x_{>}$with $f_{A}\left(x_{>}\right) \geq f_{A}(x)$ and analogously there exists an adjacent sampling point $x_{\leq}$with $f_{A}\left(x_{\leq}\right) \leq f_{A}(x)$.

Since the class of possible point spread functions is very general, this sampling theorem can be applied to much more practical applications than previous ones. Unfortunately the restriction $p<r^{\prime}$ is very strict. We conjecture that the theorem is true for any $p$ with $p+r^{\prime}<r$, but we were up to now not able to prove this formally.

\section{Conclusions}

We proved a sampling theorem which can be summarized in an extremely simple statement: By using a $p$-PSF and a square grid, which is an $r^{\prime}$-grid (with $r^{\prime}>p$ ), we can digitize any $r$-regular binary image without any topological changes if only $r>r^{\prime}+p$. This is true for any threshold value used for binarization.

Realistic cameras have very complicated point spread functions and often one does not know the exact PSF. Due to our result one does not have to know this, if only one can assume that it is nonnegative, radially symmetric and has a bounded 
support of known (or estimated) radius. Thus our result can be applied to real camera acquisition systems much better than the findings of Latecki et al. [2,3] and some of our previous papers [1,7], where only point spread functions were allowed which are constant in their whole support. Unfortunately our proof has a restriction to the maximal size of the PSF relatively to the sampling density. We think that this is not necessary and conjecture that our results can be generalized to any $p$-PSF with $r>p+r^{\prime}$. Additionally we think that equivalent theorems can be shown for other sampling grids like hexagonal or even irregular grids, but we cannot prove this yet.

\section{References}

1. Köthe, U., Stelldinger, P.: Shape Preserving Digitization of Ideal and Blurred Binary Shapes. In: I. Nyström et al. (Eds.): DGCI 2003, LNCS 2886, pp. 82-91, Springer, 2003.

2. Latecki, L.J., Conrad, C., Gross, A.: Preserving Topology by a Digitization Process. Journal of Mathematical Imaging and Vision 8, pp. 131-159, 1998.

3. Latecki, L.J.: Discrete Representation of Spatial Objects in Computer Vision. Kluwer, 1998.

4. Pavlidis, T.: Algorithms for Graphics and Image Processing. Computer Science Press, 1982.

5. Ronse, C., Tajine, M.: Morphological Sampling of Closed Sets. Image Analysis and Stereology 23, pp. 89-109, 2004.

6. Serra, J.: Image Analysis and Mathematical Morphology. Academic Press, 1982.

7. Stelldinger, P., Köthe U.: Shape Preservation During Digitization: Tight Bounds Based on the Morphing Distance. In: B. Michaelis, G. Krel (Eds.): Pattern Recognition, LNCS 2781, pp. 108-115, Springer, 2003. 